\title{
Analyse der Literatur zu Klimawirkungen in Deutschland: ein Gesamtbild mit Lücken
}

Mark Fleischhauer, Stefan Greiving, Christian Lindner, Johannes Lückenkötter, Inke Schauser

27.1 Die Studie des Netzwerks Vulnerabilität - 278

27.1.1 Auswertung nach Handlungsfeldern - 278

27.1.2 Auswertung der inhaltlichen Aussagen zu den verschiedenen Handlungsfeldern - 280

27.1.3 Methodische Ansätze der Vulnerabilitätsstudien - 283

27.1.4 Zusammenfassung - 284

$27.2 \quad$ Kurz gesagt -284

Literatur - 285 
Dieses Unterkapitel präsentiert Ergebnisse einer umfassenden Literaturauswertung zu relevanten Klimawirkungen für Deutschland, die im Rahmen des Projekts „Netzwerk Vulnerabilität“ vorgenommen wurde. In diesem Netzwerk sind 16 Bundesbehörden und Bundesinstitute sowie ein wissenschaftliches Konsortium vertreten (Umweltbundesamt 2012). Es sollte an dieser Stelle betont werden, dass aus Platzgründen viele Details nicht dargestellt werden können und daher auf die Ergebnisse der Gesamtstudie verwiesen wird (adelphi et al. 2015).

Die nach Regionen und Handlungsfeldern unterschiedlichen Auswirkungen des Klimawandels in Deutschland (Teil III) erfordern unterschiedliche Anpassungsmaßnahmen (Teil V). Sektorenübergreifende Bewertungen der vielfältigen Klimawirkungen können Entscheidungsträger dabei unterstützen, diejenigen Regionen und Themen zu identifizieren, die voraussichtlich besonders stark vom Klimawandel beeinträchtigt werden, d. h. vulnerabel sind, und wo daher vordringlich Anpassungsaktivitäten erfolgen sollten. Klimawirkungsanalysen können aber auch noch anderen Zwecken dienen (Füssel und Klein 2006; Hinkel 2011): der Vertiefung des Wissens über Klimawandelfolgen inklusive ihrer Wechselwirkungen, der Feststellung ihres Schadenspotenzials, der Erzeugung von Aufmerksamkeit für die potenziellen Gefahren des Klimawandels und der Beobachtung zeitlicher Entwicklungen von Klimawirkungen und Anpassungsaktivitäten.

Die zentrale Herausforderung für alle Klimawirkungs- und Vulnerabilitätsstudien ist es, mit der Abschätzung von den mit zukünftigen Entwicklungen verbundenen Unsicherheiten umzugehen, die von verschiedenen Quellen ausgehen, wie von bezifferbaren Fehlern in Daten über mehrdeutig formulierte Konzepte und Terminologien bis hin zu unsicheren Projektionen über menschliches Verhalten und gesellschaftliche Entwicklung (Birkmann et al. 2013). Die unsicheren künftigen Entwicklungen des Klimasignals (Klimaprojektionen), der Flächennutzungen und der sozioökonomischen Faktoren (z. B. Bevölkerungs- und Wirtschaftsentwicklung) werden aber bei fachlichen/politischen Entscheidungen von Akteuren im Abschätzungsprozess je nach zweckmäßigen fachlichen, oft aber auch politischen Grundeinstellungen unterschiedlich interpretiert. Die Verwendung von Ensembles in Klimawirkungs- und Vulnerabilitätsanalysen vergrößert die Bandbreite der Analyseergebnisse und macht somit die bestehenden Unsicherheiten sichtbar. Letztlich enthebt es die Entscheidungsträger jedoch nicht der Pflicht, aus dieser Bandbreite der Ergebnisse das für die Entscheidung von Maßnahmen geeignete auszuwählen.

Für Deutschland gab das Umweltbundesamt 2005 eine erste handlungsfeldübergreifende Analyse der Vulnerabilität in Auftrag (Zebisch et al. 2005). Seitdem wurde in Deutschland von den Behörden der Länder und des Bundes eine große Anzahl von Klimawirkungs- und Vulnerabilitätsstudien beauftragt und bearbeitet. Diese liegen entweder in Form landesweiter Studien vor, die mehrere Handlungsfelder abdecken wie z. B. für Brandenburg (Gerstengarbe et al. 2003), Baden-Württemberg (Stock 2005) oder Nordrhein-Westfalen (Kropp et al. 2009), oder auch als handlungsfeldbezogene und räumliche Teilstudien wie beispielsweise in Baden-Württemberg (LUBW 2014), Hessen (HLUG 2013) oder Rheinland-Pfalz (MWKEL 2014). Im Rahmen von Forschungsprojekten wurden ebenfalls auf europäischer (ESPON
Climate 2011; EEA 2012), deutscher (BMVBS 2010) und auf regionaler Ebene - KlimaMORO (BMVBS 2013) oder KLIMZUG (BMBF 2014) - solche Untersuchungen durchgeführt. Darüber hinaus existieren Fallbeispiele im Rahmen von Projekten des 7. Forschungsrahmenprogramms oder von INTERREG-Projekten (Projektdatenbank siehe CLIMATE-ADAPT 2014).

\subsection{Die Studie des Netzwerks Vulnerabilität}

Von 2011 bis 2015 führte das Netzwerk Vulnerabilität eine aktuelle, methodisch konsistente deutschlandweite Zusammenschau bereits bestehender Vulnerabilitätsanalysen für die Fortschreibung der Deutschen Anpassungsstrategie durch, um die heute und in Zukunft für Deutschland wichtigsten Klimawirkungen handlungsfeldübergreifend zu identifizieren (adelphi et al. 2015; Greiving et al. 2015). Die vorhandene Literatur zu Klimawirkungen und Vulnerabilitäten wurde im Hinblick auf Angaben zu Exposition, Sensitivität und Anpassungskapazität sowie Vulnerabilitätskonzepten und Bewertungsmethoden analysiert. Basierend auf dieser umfassenden Literaturauswertung erarbeitete man einen „Klimastudienkatalog“, eine interaktive Plattform, die es ermöglicht, sich strukturiert über die erwarteten Klimawirkungen in Deutschland zu informieren (Umweltbundesamt 2014). Im Folgenden präsentieren die Autoren die Ergebnisse der Literaturauswertung, wobei sie erst einen Überblick über die abgedeckten Handlungsfelder und Aussagen geben und dann die Methodik in den Studien diskutieren.

Insgesamt konnten 155 Studien ermittelt werden, von denen 75 Studien räumlich konkrete Aussagen zu Klimawirkungen in Deutschland enthalten (Stichtag: 31.08.2012). Der Großteil der Studien ist seit 2005 fertig gestellt worden, mit einem vorläufigen Maximum im Jahr 2011.

Etwa ein Drittel der untersuchten Studien (45 von 155 Studien) wurde von den Ländern (Landesministerien, Landesämter), weitere 27 Studien wurden vom Bund (Bundesministerien, Bundesämter) in Auftrag gegeben und betrachten somit die Gesamtfläche oder Teilflächen eines Bundeslandes, sodass von den 155 auf methodische Fragen untersuchten Studien 75 Studien einen klaren räumlichen Fokus auf einzelne oder mehrere Bundesländer haben.

\subsubsection{Auswertung nach Handlungsfeldern}

Die Deutsche Anpassungsstrategie DAS (Bundesregierung 2008) nennt 15 Handlungsfelder der deutschen Anpassungspolitik, darunter zwei Querschnittsthemen. Diese Untergliederung diente bei der Auswertung der in den Klimastudienkatalog des Netzwerks Vulnerabilität eingestellten 155 Studien als Grundlage für die inhaltliche Zuordnung (das DAS-Handlungsfeld Wasserhaushalt, Wasserwirtschaft, Küsten- und Meeresschutz wurde hierbei in zwei Handlungsfelder geteilt). Die Mehrheit der untersuchten Studien (92 von 155) betrachtet nicht nur ein, sondern mehrere Handlungsfelder und ist somit als „handlungsfeld- oder sektorübergreifend“ zu bezeichnen, was jedoch nicht mit einer Aggregation bzw. Integration der Analyseergebnisse über die Handlungs- 


\section{Häufigkeit untersuchter DAS-Handlungsfelder}

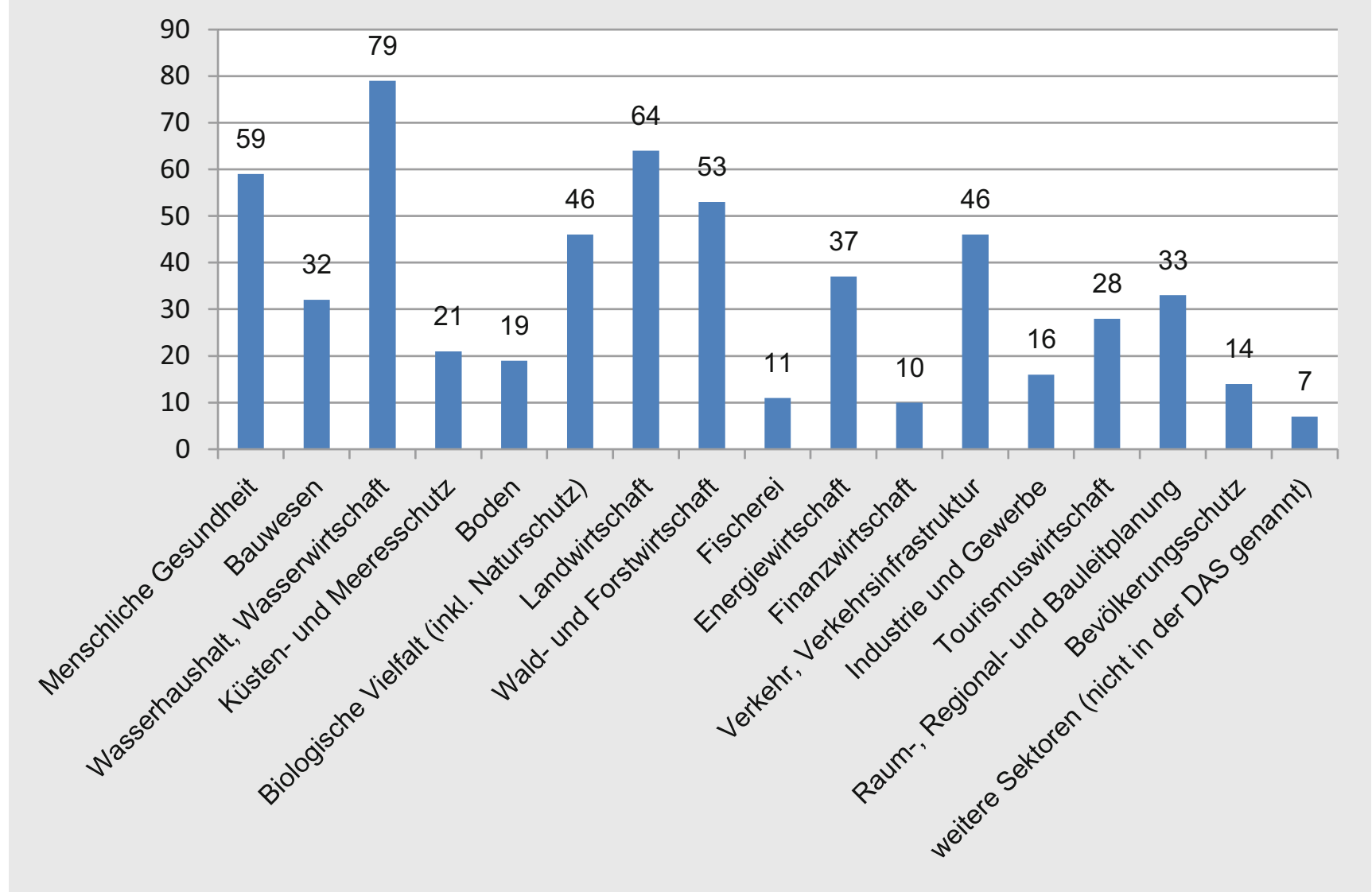

- Abb. 27.1 Anzahl untersuchter Handlungsfelder in den Klimawirkungs- und Vulnerabilitätsstudien. (adelphi et al. 2015)

felder hinweg gleichzusetzen ist. 59 Studien hingegen beschränken sich auf Aussagen zu einem einzigen Handlungsfeld der Deutschen Anpassungsstrategie (- Abb. 27.1). Die meisten Studien beschäftigen sich mit den Handlungsfeldern Wasserhaushalt und Wasserwirtschaft, Landwirtschaft, Menschliche Gesundheit sowie Forstwirtschaft. Am wenigsten gibt es zu den Handlungsfeldern Fischerei sowie Finanzwirtschaft. Die unausgewogene Verteilung fällt auf. Einerseits gibt sie einen Hinweis auf die Schwerpunkte der bisherigen Klimafolgenforschung und die Interessen der beauftragenden Institutionen, andererseits ist sie sicher auch auf die unterschiedlichen Umfänge der Handlungsfelder zurückzuführen.

Die Auswahl der untersuchten Handlungsfelder in Klimawirkungs- und Vulnerabilitätsstudien folgt keinem definierten Standard, sondern hängt von unterschiedlichen Faktoren ab, etwa von den vermuteten Auswirkungen innerhalb eines Bezugsraums (was u.a. durch die naturräumliche Ausstattung eines Raums bestimmt wird), dem zur Verfügung stehenden Budget und Zeitrahmen für die Erarbeitung der Studie, von der Datenverfügbarkeit, der Expertise der Bearbeiter und von der Interessenlage der Auftraggeber.

Nicht alle ausgewerteten Studien enthalten konkrete Ergebnisse für klar definierte Regionen, sondern einige treffen allgemeine Aussagen für Deutschland oder Europa insgesamt bzw. setzen sich in erster Linie mit methodischen Fragen zur Ermittlung von Klimawirkungen in einem bestimmten Handlungsfeld auseinander. Aus deutscher Sicht interessant ist aber vor allem, welche räumlich konkreten Aussagen sich in Bezug auf einzelne Handlungsfelder für Deutschland insgesamt sowie für die Teilregionen (hier: Bundesländer) in den Klimawirkungs- und Vulnerabilitätsstudien finden. Dies ermöglicht einen Überblick über die tatsächlich für Deutschland bzw. die deutschen Bundesländer vorliegenden Informationen und zeigt bestehende Lücken auf.

Dazu wurden ausschließlich diejenigen Studien ausgewählt, in denen konkrete raumbezogene Aussagen zu den Auswirkungen des Klimawandels in Deutschland getroffen wurden. Die für die deutschen Bundesländer sowie Deutschland insgesamt relevanten Aussagen wurden dann aus den vorliegenden Studien extrahiert und zu einer Datenbank mit Aussagen zur Klimawirkung auf Länder- und Handlungsfeldebene zusammengefasst.

Über $50 \%$ aller Aussagen zu Klimafolgen wurden in den vier Handlungsfeldern Wasserhaushalt und Wasserwirtschaft, Landwirtschaft, Wald- und Forstwirtschaft sowie Biologische Vielfalt getroffen (adelphi et al. 2015, - Abb. 27.4). Da bei diesen Handlungsfeldern ein enger Bezug zum Klimasignal besteht (insbesondere Temperatur, Niederschlag), kann hier eine starke Betroffenheit durch den Klimawandel vermutet werden. Ein weiterer Grund für den hohen Anteil dieser Handlungsfelder bei der Betrachtung in Klimawirkungs- und Vulnerabilitätsstudien besteht darin, dass es sich bei diesen Handlungsfeldern um Bereiche handelt, die für alle Bundesländer relevant sind. Ein weiterer 
Grund mag darin liegen, dass sich in diesen Handlungsfeldern aufgrund des genannten direkten Zusammenhangs die sich ändernden Klimaparameter relativ einfach in bereits bestehende Analysemodelle und -methoden einspeisen lassen. Dies gilt auch für das Handlungsfeld Menschliche Gesundheit, für das ebenfalls eine hohe Anzahl an Aussagen getroffen wurde. Hier wurde in erster Linie das Thema des Hitzeinseleffekts in großen Agglomerationsräumen untersucht (adelphi et al. 2015, - Tab. 27.1), bei dem ebenfalls ein direkter Zusammenhang zu dem sich ändernden Klima gesehen wird. Gleichwohl bestehen hier auch verstärkt indirekte Zusammenhänge, insbesondere in Bezug auf die Folgen von klimabezogenen Naturgefahren.

In den fünf Handlungsfeldern Energiewirtschaft, Tourismuswirtschaft, Boden, Verkehr, Verkehrsinfrastruktur sowie Küstenund Meeresschutz existiert nur eine relativ geringe Anzahl an Aussagen, was zum einen daran liegen könnte, dass in einem Teil dieser Handlungsfelder die Klimafolgen als nicht so gravierend eingeschätzt werden. Zum anderen liegt es aber auch daran, dass die Handlungsfelder nur für bestimmte Teilbereiche Deutschlands relevant sind, so etwa der Küsten- und Meeresschutz für die Küstenländer oder die Tourismuswirtschaft für die bedeutendsten Touristendestinationen. Schließlich sind die Zusammenhänge mit dem Klima in diesen Handlungsfeldern häufig indirekter Natur und in starkem Maße von sozioökonomischen Faktoren abhängig, d.h., die Klimafolgen werden hier insbesondere auch von der Sensitivität des Handlungsfeldes bestimmt. Da eine derartige Analyse einen höheren methodischen Aufwand erfordert, ist dies möglicherweise ein weiterer Grund, warum diese Handlungsfelder nur mit mittlerer Häufigkeit betrachtet werden.

Für eine Gruppe von vier Handlungsfeldern, nämlich Bauwesen, Fischereiwirtschaft, Industrie \& Gewerbe sowie Finanzwirtschaft, sind nur sehr wenige Aussagen in den ausgewerteten Vulnerabilitätsstudien zu finden. Als Grund könnte man vermuten, dass nur geringe Auswirkungen erwartet werden. Darüber hinaus wird bei den Akteuren in diesen Handlungsfeldern die eigene Betroffenheit durch den Klimawandel wohl noch nicht als sehr groß eingeschätzt. Bei der Fischereiwirtschaft kommt hinzu, dass dieses Handlungsfeld volkswirtschaftlich nur einen geringen Anteil ausmacht und es neben den Küstenländern auf relativ wenige Fischereibetriebe an Binnengewässern bezogen ist.

Um näherungsweise eine Aussage darüber zu machen, welche Bundesländer welche Vulnerabilitätsstudien angefertigt haben und was es für Gesamtdeutschland gibt, wurden die Aussagen in den Studien den einzelnen Bundesländern zugeordnet. Um die Übersichtlichkeit zu erhöhen, wurden die Handlungsfelder der Deutschen Anpassungsstrategie zu fünf Clustern zusammengefasst: Cluster 1: Umwelt und primärer Sektor, Cluster 2: Wasser und Fischerei, Cluster 3: Siedlungsentwicklung und Verkehr, Cluster 4: Produktion und Dienstleistung, Cluster 5: Gesundheit und Bevölkerungsschutz. D Abb. 27.2 zeigt, wo die einzelnen Bundesländer die Schwerpunkte bei ihren Studien gesetzt haben. Hier zeigt sich, dass bei einigen Bundesländern bestimmte Cluster stärker im Vordergrund stehen als bei anderen (z. B. Dienstleistung und Produktion in Nordrhein-Westfalen), häufig aber auch ganze Cluster in einigen Ländern bislang gar nicht untersucht worden sind (beispielsweise fehlt das Cluster Siedlungsentwicklung und Verkehr in sechs Bundesländern).

\subsubsection{Auswertung der inhaltlichen Aussagen zu den verschiedenen Handlungsfeldern}

Die aus den Studien extrahierten Aussagen wurden für die Literaturanalyse darüber hinaus inhaltlich ausgewertet, um eine Übersicht zum gegenwärtigen Wissensstand zur Betroffenheit Gesamtdeutschlands bzw. einzelner Bundesländer durch den Klimawandel erstellen zu können. So wurden u. a. alle Aussagen nach einem Codierleitfaden für die qualitative Bewertung der Informationen den folgenden Kategorien zugeordnet (adelphi et al. 2015):

- Starke negative Auswirkungen

- Moderate negative Auswirkungen

- Geringe negative Auswirkungen

- Positive Auswirkungen

- Hohe Unsicherheit bzw. Schwierigkeit bei der Einschätzung der Aussagen

Durch diese Bewertung der Aussagen kann für jedes Bundesland und für die gesamte Bundesrepublik ein farblich an die Darstellung einer Ampel angelehntes „Klimawirkungsdiagramm“ generiert werden, das alle vorliegenden Aussagen zu einem Handlungsfeld oder zu allen Handlungsfeldern in einem Bundesland nebeneinander abbildet und in gewissem Rahmen vergleichbar macht (adelphi et al. 2015, - Abb. 27.3).

- Abb. 27.4 nennt anhand der einzelnen DAS-Handlungsfelder die Anzahl der Aussagen zu erwarteten Auswirkungen sowie die Anzahl der Aussagen, die laut den entsprechenden Studien explizit mit einer deutlichen Unsicherheit bzw. Schwierigkeit der Bewertung verbunden sind (z. B. Aussagen wie "auf vielfältige Weise beeinflusst“" „wirken sich in unterschiedlicher Form und Intensität aus“, „lassen sich nur sehr schwer Trends feststellen"). Davon ausgehend, dass bei der Untersuchung eines Handlungsfeldes innerhalb einer Klimawirkungs- und Vulnerabilitätsstudie jeweils ein methodischer Ansatz gewählt wurde, mit dem sowohl positive als auch negative Auswirkungen bestimmt werden können, kann man für diese Gegenüberstellung davon ausgehen, dass der Vergleich zwischen negativen und positiven Auswirkungen für ein Handlungsfeld tendenziell anzeigt, ob in diesem Handlungsfeld ausschließlich Nachteile oder ob in einem gewissen Maß auch positive Auswirkungen zu erwarten sind. Dieser Vergleich ist jedoch lediglich für die Betrachtung innerhalb eines Handlungsfeldes zulässig. Eine breite Streuung positiver und negativer Auswirkungen kann ein Hinweis darauf sein, dass unterschiedliche Studien auch zu unterschiedlichen Ergebnissen kommen können, etwa weil einige Analysen auf Klimatrends, andere auf Extremereignissen aufbauen, neue Daten oder Modelle zur Verfügung stehen oder unterschiedliche Modellensembles gewählt wurden.

Um ein präziseres Bild von Forschungsschwerpunkten und -lücken zu erhalten, werteten die Autoren im Auftrag des Umweltbundesamtes (adelphi et al. 2015) die Literatur auch hinsichtlich einzelner Klimawirkungen und ihrer Zuordnung zu den jeweiligen Handlungsfeldern der Deutschen Anpassungsstrategie DAS (Bundesregierung 2008) aus (• Tab. 27.1).

Die am häufigsten untersuchten Klimawirkungen ( $\bullet$ Tab. 27.1) gehören zu den Handlungsfeldern, die auch ihrerseits am häu- 
Tab. 27.1 Liste der am häufigsten untersuchten Klimawirkungen. (adelphi et al. 2015)

\begin{tabular}{|c|c|c|}
\hline DAS-Handlungsfeld & Klimawirkung & $\begin{array}{l}\text { Anzahl der Unter- } \\
\text { suchungen }\end{array}$ \\
\hline Wasserhaushalt, -wirtschaft & Hochwasser (alle Typen) & 27 \\
\hline Landwirtschaft & Ertrag & 25 \\
\hline Wasserhaushalt, -wirtschaft & Grundwasserstand (Quantität) & 24 \\
\hline Menschliche Gesundheit & Hitzestress (z. B. Herz- Kreislauf-Erkrankungen, Hitzetote, Leistungsfähigkeit) & 23 \\
\hline Biologische Vielfalt & Veränderung von Biotopen/Habitaten & 21 \\
\hline Landwirtschaft & Verschiebung agrophänologischer Phasen und Veränderung der Wachstumsperiode & 19 \\
\hline Wald- und Forstwirtschaft & Veränderung von Nutzfunktionen (Holzproduktion) & 19 \\
\hline \multirow[t]{2}{*}{ Biologische Vielfalt } & Verschiebung von Ökosystemarealen & 18 \\
\hline & Veränderung phänologischer Phasen (inkl. Früh- und Spätfröste) & 17 \\
\hline \multirow[t]{3}{*}{ Wald- und Forstwirtschaft } & Hitze- und Trockenstress & 17 \\
\hline & Schäden durch Extremereignisse (vor allem Windwurf) & 17 \\
\hline & Schädlinge - trocken (Insekten) & 17 \\
\hline Biologische Vielfalt & Veränderung der Ökosystemdienstleistungen & 16 \\
\hline \multirow[t]{3}{*}{ Boden } & Erosion (fluvial, äolisch)/Bodenverdichtung/Hangrutschung & 15 \\
\hline & Veränderung Bodenwassergehalt, Grundwasserneubildung & 15 \\
\hline & Veränderung Produktionsfunktionen (Standortstabilität, Bodenfruchtbarkeit) & 15 \\
\hline Biologische Vielfalt & Verbreitung invasiver Arten & 15 \\
\hline Energiewirtschaft & Verfügbarkeit von Kühlwasser für thermische Kraftwerke $\rightarrow$ veränderte Leistung & 15 \\
\hline Landwirtschaft & Schäden durch Extremereignisse & 15 \\
\hline Wald- und Forstwirtschaft & Waldbrand & 15 \\
\hline \multirow[t]{2}{*}{ Wasserhaushalt, -wirtschaft } & Niedrigwasser & 15 \\
\hline & Quantität Oberflächenwasser & 15 \\
\hline Landwirtschaft & Vernässung, Trocken- und Frostschäden, Wechselfröste & 14 \\
\hline Tourismuswirtschaft & Beeinträchtigung/Wegfall touristischer Angebote & 14 \\
\hline Wasserhaushalt, -wirtschaft & Grundwasserverfügbarkeit & 14 \\
\hline Boden & Veränderung Nährstoffspeicherfunktionen (Kohlenstoff, Nitrat etc.) & 13 \\
\hline Menschliche Gesundheit & Überträger von Krankheitserregern & 13 \\
\hline Tourismuswirtschaft & Saisonale Nachfrageverschiebung & 13 \\
\hline Wald- und Forstwirtschaft & Veränderung der Baumartenzusammensetzung & 13 \\
\hline Biologische Vielfalt & Aussterben von Arten & 12 \\
\hline Menschliche Gesundheit & Allergische Reaktionen (pflanzlich und tierisch) & 12 \\
\hline Wasserhaushalt, -wirtschaft & Durchfluss Oberflächenwasser (jährlich, saisonal, täglich) & 12 \\
\hline Boden & Veränderung Filter-/Pufferfunktionen (Wasser, Schadstoffe etc.) & 11 \\
\hline Biologische Vielfalt & Rückgang der Bestände & 11 \\
\hline Landwirtschaft & Schädlinge & 11 \\
\hline Verkehr, Verkehrsinfrastruktur & Schiffbarkeit von Binnenwasserstraßen & 11 \\
\hline Wald- und Forstwirtschaft & Veränderung von Schutzfunktionen (Naturgefahren, $\mathrm{CO}_{2}$-Sequestrierung) & 10 \\
\hline Wasserhaushalt, -wirtschaft & Trinkwasser & 10 \\
\hline
\end{tabular}




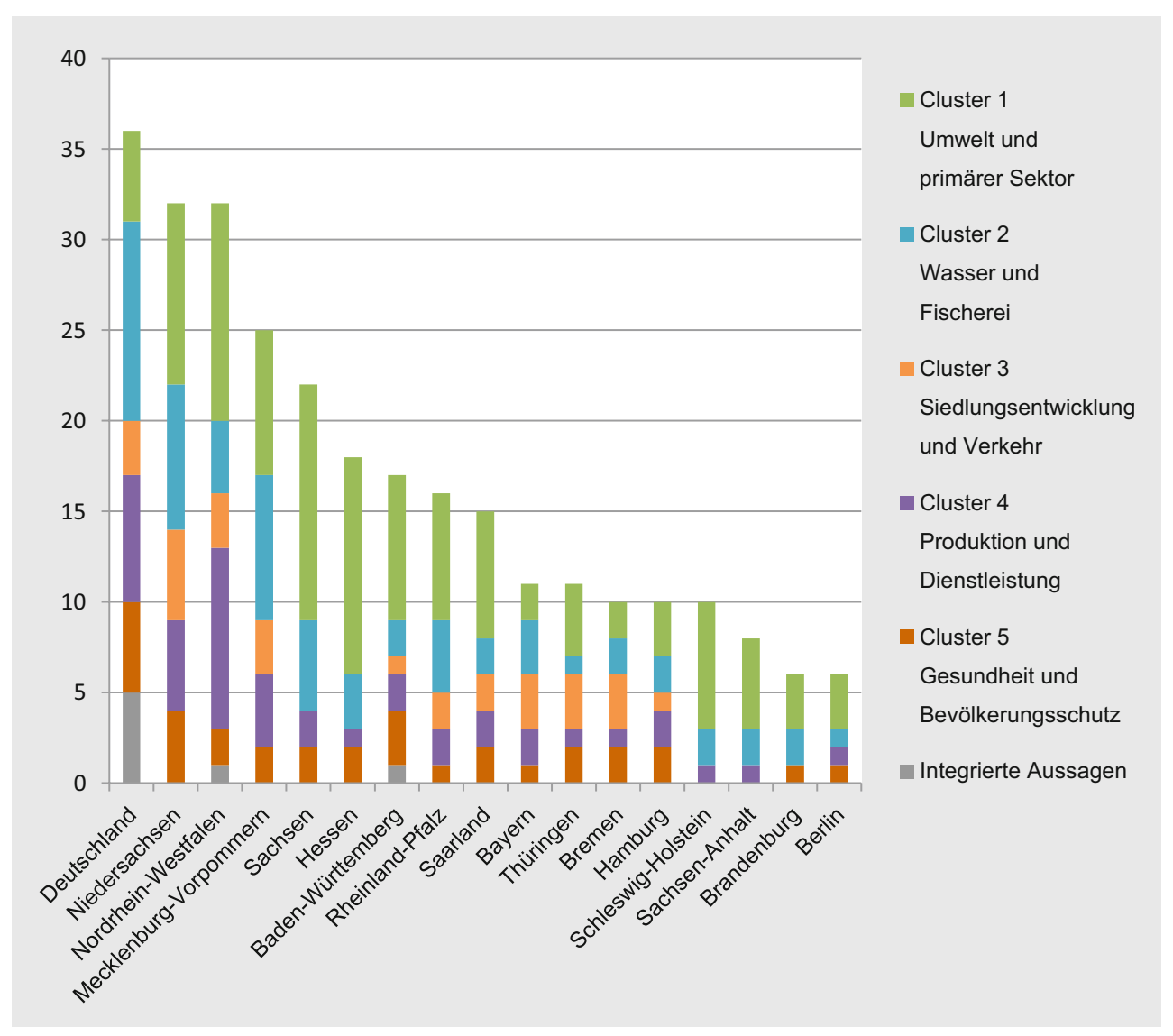

Kategorien

\section{Klimawirkungsdiagramm}

\begin{tabular}{|l|l|}
\hline $\begin{array}{l}\text { starke negative } \\
\text { Auswirkungen } \\
\text { + Anzahl der } \\
\text { Aussagen }\end{array}$ & $\begin{array}{l}\text { positive } \\
\text { Auswirkungen } \\
\text { + Anzahl der } \\
\text { Aussagen }\end{array}$ \\
\hline $\begin{array}{l}\text { mittlere negative } \\
\text { Auswirkungen } \\
\text { + Anzahl der } \\
\text { Aussagen }\end{array}$ & $\begin{array}{l}\text { Auswirkungen } \\
\text { + Anzahl der } \\
\text { Aussagen }\end{array}$ \\
\hline $\begin{array}{l}\text { geringe negative } \\
\text { Auswirkungen } \\
\text { + Anzahl der } \\
\text { Aussagen }\end{array}$ & $\begin{array}{l}\text { Gesamtzahl der } \\
\text { Studien }\end{array}$ \\
\hline
\end{tabular}

Die Darstellungsweise ermöglicht es, auch bei widersprüchlichen Angaben zu einem Sektor in einem Bundesland diese Ausprägungen darzustellen.

Dargestellt werden:

1. Stärke und Richtung der Auswirkungen (rot, orange, gelb, blau, grün)

2. Anzahl der Aussagen, die zur jeweiligen Ausprägung gemacht werden (Zahl)

3. Gesamtzahl der Studien (grau, Zahl)

\section{Beispieldarstellung Sektor Landwirtschaft}

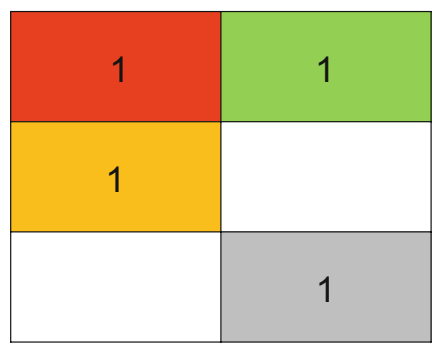

„Die sich abzeichnenden Klimaänderungen wirken sich in unterschiedlicher Form und Intensität auf den Ackerbau und die Grünlandbewirtschaftung, den Gartenbau und die Tierhaltung aus. Dabei ergeben sich negative, aber auch einige positive Effekte. [...] Mit der Verlängerung der Vegetationsperiode und einer zunehmenden Photosyntheserate sind - ausreichende Wasserversorgung vorausgesetzt - höhere Erträge zu erzielen. [...]

Durch ansteigende Temperatur und verstärkte

Ausbreitungsmöglichkeiten im internationalen Warenverkehr sind eine erhebliche Zunahme Wärme liebender Insekten, wie Kartoffelkäfer, Blattläuse und Maiszünsler, sowie eine Zunahme von Primärschäden durch Blattfraß oder Saugschäden und auch Qualitätsverluste zu verzeichnen. [...]

Wärme liebende Krankheiten nehmen zu, denen kurze Feuchte- oder Tauphasen zur Ausbreitung ausreichen (z.B. Getreideroste,

Setosphaerica turcica - Blattflecken an Mais, Alternaria -

Dürrfleckenkrankheit der Kartoffel, evtl. Apfelschorf, Feuerbrand).“
- Abb. 27.2 Anzahl der Aussagen aus den Klimawirkungs- und Vulnerabilitätsstudien zu den Auswirkungen des Klimawandels pro Cluster und Bundesland. (eigene Darstellung nach adelphi et al. 2015)
- Abb. 27.3 Darstellung von Handlungsfeldaussagen aus Klimastudien im Klimawirkungsdiagramm. (adelphi et al. 2015); fiktives Beispiel in Anlehnung an LWK NRW 2012 
- Abb. 27.4 Anzahl und Tendenz der Aussagen aus den Klimawirkungs- und Vulnerabilitätsstudien zu den Auswirkungen des Klimawandels. (adelphi 2015)

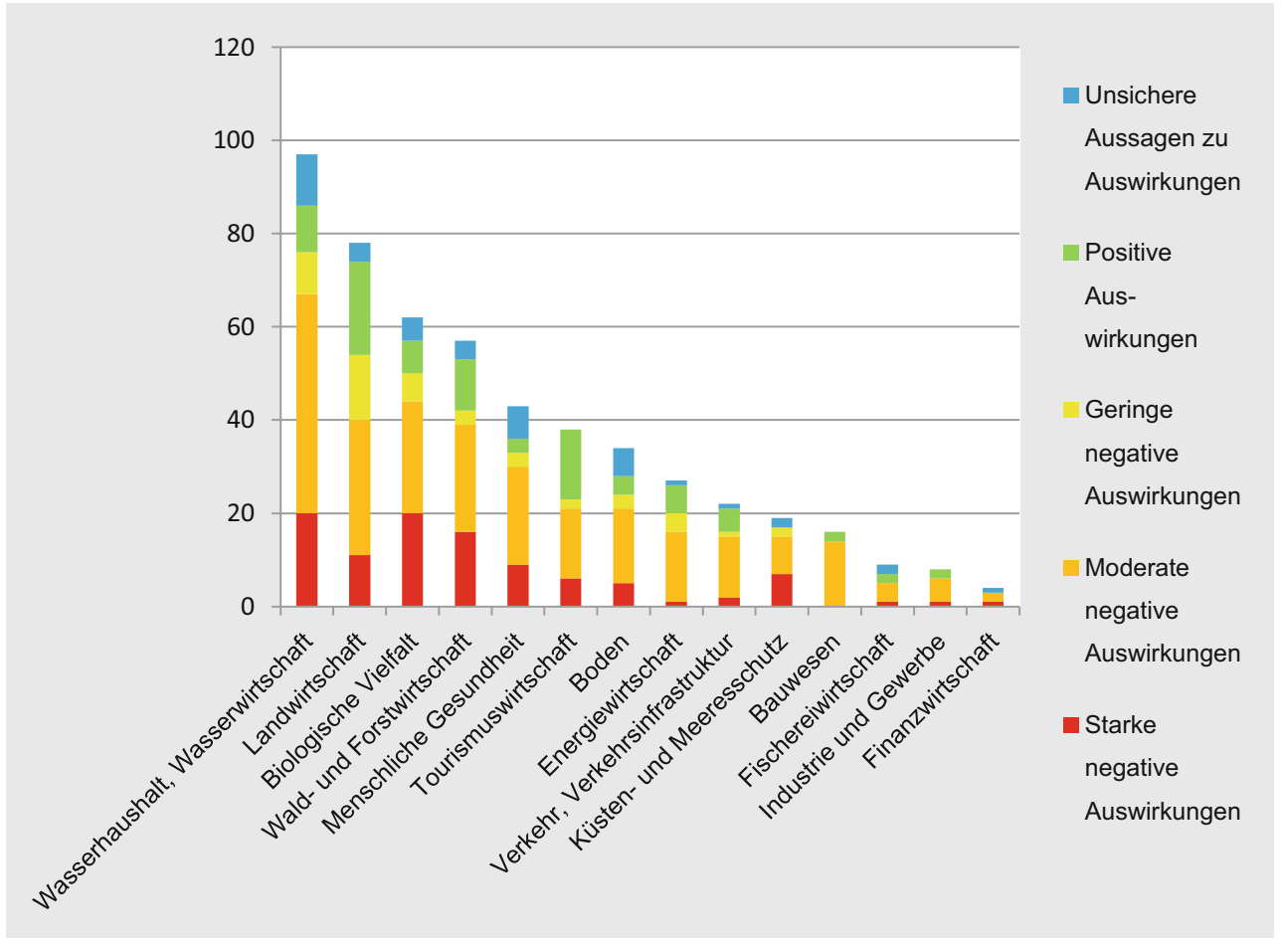

figsten in Studien untersucht wurden, also Wasserhaushalt und Wasserwirtschaft, Landwirtschaft, Menschliche Gesundheit, Biologische Vielfalt sowie Wald- und Forstwirtschaft (- Abb. 27.4). Insgesamt taucht nur die Hälfte der Handlungsfelder bei den am häufigsten untersuchten Klimawirkungen auf. Dies kann einerseits in der Bedeutung eines Handlungsfeldes begründet sein, andererseits liegt es aber möglicherweise auch daran, dass bestimmte Klimafolgen oft untersucht wurden, weil sie sich einfach untersuchen lassen (Bestehen geeigneter Modelle, Datenverfügbarkeit), ungeachtet der Tatsache, ob sie tatsächlich ein sehr bedeutendes Problem darstellen oder nicht.

Bei den am wenigsten untersuchten Klimafolgen zeigt sich, dass insbesondere in den eher technischen, infrastrukturellen Handlungsfeldern (Bauwesen, Energiewirtschaft, Tourismus und Verkehr, Verkehrsinfrastruktur) noch Lücken in der Forschung bestehen. Ein wesentlicher Grund ist vermutlich, dass die hier genannten Auswirkungen eher indirekte Klimafolgen darstellen und daher weniger gut abgeschätzt werden können, andererseits aber vonseiten der Experten aus handlungsfeldübergreifender Perspektive als bedeutend im Vergleich auch zu anderen Klimafolgen eingeschätzt werden (adelphi et al. 2015). Insgesamt zeigt sich daraus, dass die in der Deutschen Anpassungsstrategie genannten Handlungsfelder in der Analyse der Klimawirkungen also letztlich in der aktuellen Klimafolgenforschungslandschaft sehr ungleich repräsentiert sind.

\subsubsection{Methodische Ansätze der Vulnerabilitätsstudien}

Eine grundsätzliche Unterscheidung der Untersuchungsansätze zur Abschätzung von Klimafolgen ergibt sich bereits beim Grundverständnis. Hier steht auf der einen Seite das vom IPCC in der Vergangenheit verwendete Konzept zur Abschätzung der Klimawandelvulnerabilität (IPCC 2001, 2007; - Kap. 26), das in rund $40 \%$ der Studien (66 von 155) Verwendung findet. Es betrachtet die Verwundbarkeit eines Systems als Funktion seiner Exposition gegenüber einem Klimasignal, seiner Sensitivität gegenüber diesem Klimasignal und seiner potenziellen Anpassungskapazität. Auf der anderen Seite steht in erster Linie das später auch im Fünften IPCC-Sachstandsbericht verwendete Risikokonzept (IPCC 2014), das in etwa einem Viertel der Studien (37 von 155) zur Anwendung kommt. Hierbei wird zwischen den das Risiko determinierenden Faktoren (Exposition, Vulnerabilität und Gefahrenkomponente) unterschieden, also eine sogenannte Risikoperspektive eingenommen ( Kap. 26).

In den restlichen Studien finden sich Mischformen aus beiden Ansätzen bzw. gänzlich andere Konzepte, teilweise führen die Autoren gar nicht aus, wie der konzeptionelle Zusammenhang zwischen Klimaänderungen und den betroffenen Handlungsfeldern zu verstehen ist (- Abb. 27.5).

In den ausgewerteten Klimawirkungs- und Vulnerabilitätsstudien kamen unterschiedliche methodische Bewertungsansätze zum Einsatz: Bewertungen können rein qualitativ sein, d.h., sie verzichten gänzlich auf die „Rechenbarkeit“ der Informationen und beschreiben verbal. Die Bewertung erfolgt beispielsweise als Einschätzung „hoch“, „mittel“, „gering“. Sie können sich jedoch auch auf semiquantitative Bewertungsverfahren stützen. Bei diesen Verfahren erfolgt die Gewinnung der Daten nach qualitativen Kriterien, d.h., nicht direkt in Zahlen darstellbare Sachverhalte werden auf Basis subjektiver Bewertungen, etwa nach Kosten, Nutzen oder Punktwerten, in rechenbare Zahlenwerte übersetzt (etwa mittels einer Nutzwertanalyse). Schließlich kann die Bewertung quantitativ sein und auf Indikatoren fußen, die z. B. aus Messdaten oder durch Modellrechnungen ermittelt werden. Da die meisten dieser Analysen sich mit der Zukunft befassen, sind 


\section{Konzeptioneller Ansatz}

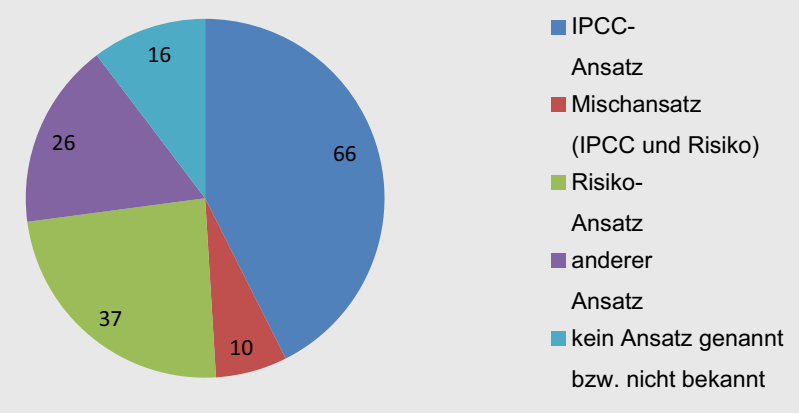

- Abb. 27.5 Konzeptionelle Ansätze in den ausgewerteten Klimawirkungsund Vulnerabilitätsstudien. (adelphi et al. 2015)

Daten aus Klimaprojektionen, aber auch sozioökonomische Szenarien, die zukünftige Zustände unserer Gesellschaft darstellen, notwendige Voraussetzung.

\subsubsection{Zusammenfassung}

Die betrachteten Zeiträume in den ausgewerteten Vulnerabilitäts- und Klimawirkungsstudien variieren, sie reichen in den früheren Studien bis 2055, haben jedoch einen Schwerpunkt beim Zeitraum 2071-2100. Für die Politikberatung günstig sind Studien, die zwischen einer nahen (z. B. 2035) und einer fernen Zukunft (z. B. 2085) unterscheiden. Mit wenigen Ausnahmen, etwa ESPON Climate (ESPON Climate 2011) und KlimaMORO-Vorstudie (BMVBS 2010), werden keine integrierten Aussagen über mehrere Handlungsfelder hinweg getroffen. Ebenso spielt Anpassungskapazität - neben Klimawirkungen die zweite Komponente von Klimavulnerabilität - in den meisten Studien eine nur untergeordnete Rolle und wird allenfalls qualitativ einbezogen, sodass man hier in der Regel von Klimawirkungsstudien und weniger von Vulnerabilitätsstudien sprechen muss.

Zusammenfassend hat die Auswertung von adelphi et al. (2015) gezeigt, dass innerhalb einzelner Studien oft sehr unterschiedliche Ansätze verwendet wurden. Auch die Studien selbst unterscheiden sich erheblich voneinander, was zum einen am wissenschaftlichen Fortschritt (neue Emissionsszenarien, neue Modelle), zum Teil aber auch an der Auswahl der zu untersuchenden Handlungsfelder und der betrachteten Themen liegt.

Für einige Bundesländer liegen Einzelstudien zu verschiedenen Handlungsfeldern bzw. zu verschiedenen Themen innerhalb eines Handlungsfeldes vor. Diese Studien schätzen beispielsweise für die süddeutschen Bundesländer Bayern, Baden-Württemberg und Rheinland-Pfalz die Auswirkungen des Klimawandels auf die Wasserwirtschaft ab (DWD et al. 2005). Für Sachsen-Anhalt wurde eine Studie zu den Kosten der Anpassung an den Klimawandel in Auftrag gegeben (UFZ 2011). In Hessen wurden knapp 20 Einzelstudien (zum Teil Aktualisierungen vorheriger Studien) zu den Auswirkungen des Klimawandels in verschiedenen
Handlungsfeldern (insbesondere Biologische Vielfalt, Land- und Forstwirtschaft) angefertigt (HLUG 2013). Dabei fällt auf, dass die Studien keiner einheitlichen Begriffssystematik folgen bzw. je nach Handlungsfeld unterschiedliche Definitionen verwenden, die als jeweils geeignet oder üblich gelten.

Viele Studien, die Aussagen zu den Auswirkungen des Klimawandels in Deutschland machen, beziehen sich nicht auf einzelne Bundesländer oder Teilregionen, sondern decken mit den Aussagen zu einzelnen Handlungsfeldern das gesamte Bundesgebiet ab. Als Beispiele aus den in den Literaturüberblick eingeflossenen Studien sind hier die Studien zu Auswirkungen des Klimawandels auf die Schadensituation in der deutschen Versicherungswirtschaft (GDV \& PIK 2011), die Herausforderung des Klimawandels für den Bevölkerungsschutz (BBK 2011) oder die verschiedenen Studien der BASt zu den Auswirkungen auf Transport und Verkehr zu nennen (z. B. BASt 2010). Darüber hinaus sind auch europaweite Studien zu nennen, die zum Teil handlungsfeldübergreifende (z. B. von der EEA 2012 oder ESPON Climate 2011), zum Teil aber auch auf einzelne Handlungsfelder bezogene Aussagen machen.

Fast alle Studien, die sich auf mehrere Handlungsfelder beziehen, betrachten die einzelnen Handlungsfelder nebeneinander, d.h., es erfolgt keine Integration etwa im Sinne eines Gesamtindex. In der Regel werden bei der Abschätzung der zukünftigen Auswirkungen des Klimawandels die zukünftigen Klimaänderungen mit der gegenwärtigen Sensitivität in Beziehung gesetzt. Die Entwicklung der Sensitivitäten, die einen hohen Einfluss auf zukünftige Klimawirkungen haben, und deren zukünftiger $\mathrm{Zu}$ stand wird in den ausgewerteten Studien nicht einbezogen. Diese Betrachtung war eines der Ziele bei der Analyse von Klimafolgen im Netzwerk Vulnerabilität. Daher wurde dort mit Szenarien der zukünftigen Siedlungs- und Bevölkerungsentwicklung gearbeitet (Greiving et al. 2015; adelphi et al. 2015).

Ein Vergleich der Betroffenheit der Bundesländer untereinander ist auf Basis der bestehenden Studien nicht möglich. Dafür sind die gegenwärtig vorhandenen Klimawirkungs- und Vulnerabilitätsstudien von zu großer Heterogenität: in Bezug auf Untersuchungsgegenstände, Methoden und Bewertungsmaßstäbe. Diese Grenzen der Aussagefähigkeit müssen bei der Verwendung des Klimastudienkatalogs (Umweltbundesamt 2014) und bei der Interpretation der Ergebnisse unbedingt berücksichtigt werden. Der Klimastudienkatalog ist daher als Informationssystem über bestehende Studien zu verstehen und sollte nicht als Grundlage für eine Vulnerabilitätsbewertung auf Bundesebene genutzt werden.

\subsection{Kurz gesagt}

Abschließend kann festgehalten werden, dass eine große Bandbreite an Ansätzen zur Bewertung von Vulnerabilitäten oder Klimawandelfolgen existiert, die gegenwärtig vorhandenen Klimawirkungs- und Vulnerabilitätsstudien von großer Heterogenität gekennzeichnet sind und keine vergleichbare Methodik existiert.

Darüber hinaus finden sich unterschiedliche konzeptionelle Ansätze (IPCC-Vulnerabilitätsansatz oder Risikoansatz), 


\section{Literatur}

Betrachtungszeiträume (z. B. 2050, 2085, 2100), verschiedene Klimamodelle und verschiedene Emissionsszenarien, und die Klimamodelldaten werden unterschiedlich regionalisiert. In einigen Studien werden integrierte Modelle zur Abschätzung der zukünftigen Auswirkungen eingesetzt, in anderen Studien plausible Annahmen aufgrund der Untersuchungsergebnisse rezenter Daten getroffen, sodass sich ein quantitativer Vergleich zwischen den Ergebnissen der Studien ebenso verbietet wie eine Verwendung auf anderen räumlichen Ebenen. Insbesondere bestehen in den eher technischen, infrastrukturellen Handlungsfeldern (Bauwesen, Energiewirtschaft, Tourismus und Verkehr, Verkehrsinfrastruktur) noch Lücken in der Forschung.

In der Konsequenz ist es daher nicht möglich, die Ergebnisse aus den verschiedenen Klimawirkungs- und Vulnerabilitätsstudien zu handlungsfeld- oder ländergrenzenüberschreitenden Aussagen zu aggregieren und auf dieser Basis flächendeckend vergleichende Aussagen zu den Auswirkungen und zum Anpassungsbedarf an den Klimawandel in Deutschland zu machen.

Durch die Zuordnung der Aussagen mithilfe eines Codierleitfadens kann für jedes Bundesland einzeln, aber auch für die gesamte Bundesrepublik ein „Klimawirkungsdiagramm“ generiert werden, das alle Aussagen zu einem Handlungsfeld oder zu allen Handlungsfeldern nebeneinander abbildet, wodurch die Handlungsfelder vergleichbar werden.

Ein sinnvolles Konzept für eine regionen- und handlungsfeldübergreifende Klimafolgen- bzw. Vulnerabilitätsbewertungsmethode sollte an den während der Literaturanalyse identifizierten zentralen Kritikpunkten ansetzen, wie beispielsweise einer klaren Definition der Begriffe und einem handlungsfeldübergreifenden Ansatz, der auch die Wechselwirkungen zwischen den Handlungsfeldern berücksichtigt. Einzelne Handlungsfelder oder Klimafolgen sollten nicht aufgrund von Datenengpässen oder aus arbeitsökonomischen Gründen aus den Studien herausfallen. Eine Darstellung der Ergebnisse in räumlich differenzierter Weise erleichtert die Umsetzung in Anpassungsmaßnahmen.
Adelphi, PRC, EURAC (2015) Vulnerabilität Deutschlands gegenüber dem Klimawandel. Umweltbundesamt. Clim Chang 24, Dessau-Roßlau. https:// www.umweltbundesamt.de/sites/default/files/medien/378/publikationen/climate_change_24_2015_vulnerabilitaet_deutschlands_gegenueber_dem_klimawandel_0.pdf.ZZugegriffen: 3. Jan. 2016

BASt - Bundesanstalt für Straßenwesen (2010) Anpassungsstrategie Klimawandel. Arbeitsgruppe „Klima“ der BASt. Pressemitteilung vom Dezember 2010. http://www.bast.de/DE/Presse/Downloads/2010-27-langfassungpressemitteilung.pdf?_blob=publicationFile. Zugegriffen: 11. Aug. 2016

BBK - Bundesamt für Bevölkerungsschutz und Katastrophenhilfe (2011) Klimawandel - Herausforderung für den Bevölkerungsschutz. Bundesamt für Bevölkerungsschutz und Katastrophenhilfe (BBK), Bonn

Birkmann J, Böhm HR, Buchholz F, Büscher D, Daschkeit A, Ebert S, Fleischhauer M, Frommer B, Köhler S, Kufeld W, Lenz S, Overbeck G, Schanze J, Schlipf S, Sommerfeldt P, Stock M, Vollmer M, Walkenhorst O (2013) Glossar Klimawandel und Raumentwicklung (2), überarbeitete Fassung. Hannover. E-Paper der ARL, Bd. 10.

BMBF - Bundesministerium für Bildung und Forschung (2014) KLIMZUG - Klimawandel in den Regionen zukunftsfähig gestalten. http://www.klimzug. de/. Zugegriffen: 22. Jan. 2014

BMVBS - Bundesministerium für Verkehr, Bau und Stadtentwicklung (2010) Klimawandel als Handlungsfeld der Raumordnung. BMVBS-Forschungen, Bd. 144. BMVBS, Berlin

BMVBS - Bundesministerium für Verkehr, Bau und Stadtentwicklung (2013) Wie kann Regionalplanung zur Anpassung an den Klimawandel beitragen? Ergebnisbericht des Modellvorhabens der Raumordnung "Raumentwicklungsstrategien zum Klimawandel“ (KlimaMORO). BMVBS-Forschungen, Bd. 157. BMVBS, Berlin

Bundesregierung (2008) Deutsche Anpassungsstrategie an den Klimawandel. http://www.bmub.bund.de/fileadmin/bmu-import/files/pdfs/allgemein/ application/pdf/das_gesamt_bf.pdf

CLIMATE-ADAPT - The European Climate Adaptation Platform (2014) Climate Change Adaptation in Europe. http://climate-adapt.eea.europa.eu/. Zugegriffen: 22. Jan. 2014

DWD, LUBW, LLfU \& LUWG (2005) KLIWA - Kooperationsvorhaben Klimaveränderung und Konsequenzen für die Wasserwirtschaft

EEA - European Environment Agency (2012) Climate change, impacts and vulnerability in Europe 2012. An indicator-based report. European Environment Agency, Copenhagen

ESPON Climate (2011) Climate change and territorial effects on regions and local economies in Europe. http://www.espon.eu/main/Menu_Projects/ Menu_AppliedResearch/climate.html

Füssel HM, Klein R (2006) Climate change vulnerability assessments: an evaluation of conceptual thinking. Clim Chang 75:301-329

GDV - Gesamtverband der Deutschen Versicherungswirtschaft e.V. \& PIK Potsdam-Institut für Klimafolgenforschung (2011) Auswirkungen des Klimawandels auf die Schadensituation in der deutschen Versicherungswirtschaft - Kurzfassung Hochwasser

Gerstengarbe F-W, Badeck F, Hattermann F, Krysanova F, Lahmer V, Lasch W, Stock P, Suckow M, Wechsung F, Werner PC (2003) Studie zur klimatischen Entwicklung im Land Brandenburg bis 2055 und deren Auswirkungen auf den Wasserhaushalt, die Forst- und Landwirtschaft sowie die Ableitung erster Perspektiven (Brandenburgstudie II). PIK Report, Bd. 83. Gefördert durch das Ministerium für Landwirtschaft, Umweltschutz und Raumordnung des Landes Brandenburg. Potsdam: PIK

Greiving S, Zebisch M, Schneiderbauer S, Lindner C, Lückenkötter J, Fleischhauer M, Buth M, Kahlenborn W, Schauser I (2015) A consensus based vulnerability assessment to climate change in Germany. Int J Clim Chang Strat Manage 7(3):306-326

Hinkel J (2011) Measuring vulnerability and adaptive capacity: towards a clarification of the science-policy interface. Glob Environ Chang 21(1):198-208

HLUG - Hessisches Landesamt für Umwelt und Geologie (2013) Fachzentrum Klimawandel Hessen. http://klimawandel.hlug.de/. Zugegriffen: 22. Jan. 2014

IPCC - Intergovernmental Panel on Climate Change (2001) Climate Change 2001: Synthesis Report. Contribution of the Working Group I, II, and III to 
the Third Assessment Report of the Intergovernmental Panel on Climate Change. Cambridge University Press, Cambridge

IPCC - Intergovernmental Panel on Climate Change (2007) Climate Change 2007: Synthesis Report. Contribution of Working Groups I, II and III to the Fourth Assessment Report of the Intergovernmental Panel on Climate Change. Cambridge University Press, Cambridge

IPCC - Intergovernmental Panel on Climate Change (2014) Summary for policymakers. Climate change 2014: Impacts, adaptation, and vulnerability. Part A: Global and sectoral aspects. In: Field CB, Barros VR, Dokken DJ, Mach KJ, Mastrandrea MD, Bilir TE, Chatterjee M, Ebi KL, Estrada YO, Genova RC, Girma B, Kissel ES, Levy AN, MacCracken S, Mastrandrea PR, White LL (Hrsg) Contribution of Working Group II to the Fifth Assessment Report of the Intergovernmental Panel on Climate Change. Cambridge University Press, Cambridge, S 1-32

Kropp J, Holsten A, Lissner T, Roithmeier O, Hattermann F, Huang S, Rock J, Wechsung F, Lüttger A, Pompe S, Kühn I, Costa L, Steinhäuser M, Walther C, Klaus M, Ritchie S, Metzger M (2009) Klimawandel in Nordrhein-Westfalen - Regionale Abschätzung der Anfälligkeit ausgewählter Sektoren. Abschlussbericht des Potsdam-Instituts für Klimafolgenforschung (PIK) für das Ministerium für Umwelt und Naturschutz, Landwirtschaft und Verbraucherschutz Nordrhein-Westfalen (MUNLV)

LUBW - Landesamt für Umwelt, Messungen und Naturschutz Baden-Württemberg (2014) Klimawandel und modellhafte Anpassung in BadenWürttemberg (KLIMOPASS). http://www.lubw.baden-wuerttemberg.de/ servlet/is/69206/. Zugegriffen: 22. Jan. 2014

MWKEL - Ministerium für Wirtschaft, Klimaschutz, Energie und Landesplanung Rheinland-Pfalz (2014) Rheinland-Pfalz Kompetenzzentrum für Klimawandelfolgen. http://www.klimawandel-rlp.de/. Zugegriffen: 22. Jan. 2014

Stock M (2005) Klimawandel, Auswirkungen, Risiken und Anpassung (KLARA). Gefördert von der Landesanstalt für Umweltschutz Baden-Württemberg (LfU). PIK Report, Bd. 99. Potsdam-Institut für Klimafolgenforschung, Potsdam

UFZ - Helmholtz-Zentrum für Umweltforschung (2011) Kosten der Anpassung an den Klimawandel - Eine ökonomische Analyse ausgewählter Sektoren in Sachsen-Anhalt

Umweltbundesamt (2012) Netzwerk Vulnerabilität. http://www.netzwerkvulnerabilitaet.de

Umweltbundesamt (2014) Klimastudienkatalog - Auswertung von Klimawirkungs- und Vulnerabilitätsstudien. http://netzwerk-vulnerabilitaet.de/ klimastudienkatalog/index.php?view=karte. Zugegriffen: 24. Aug. 2016

Zebisch M, Grothmann T, Schröter D, Hasse C, Fritsch U, Cramer W (2005) Klimawandel in Deutschland. Vulnerabilität und Anpassungsstrategie klimasensitiver Systeme. Umweltbundesamt, Dessau-Rosslau

Open Access Dieses Kapitel wird unter der Creative Commons Namensnennung 4.0 International Lizenz (http://creativecommons.org/ licenses/by/4.0/deed.de) veröffentlicht, welche die Nutzung, Vervielfältigung, Bearbeitung, Verbreitung und Wiedergabe in jeglichem Medium und Format erlaubt, sofern Sie den/die ursprünglichen Autor(en) und die Quelle ordnungsgemäß nennen, einen Link zur Creative Commons Lizenz beifügen und angeben, ob Änderungen vorgenommen wurden.

Etwaige Abbildungen oder sonstiges Drittmaterial unterliegen ebenfalls der genannten Creative Commons Lizenz, sofern sich aus der Abbildungslegende oder der Quellreferenz nichts anderes ergibt. Sofern solches Drittmaterial nicht unter der genannten Creative Commons Lizenz steht, ist eine Vervielfältigung, Bearbeitung oder öffentliche Wiedergabe nur mit vorheriger Zustimmung des betreffenden Rechteinhabers oder auf der Grundlage einschlägiger gesetzlicher Erlaubnisvorschriften zulässig. 